\title{
Magnetic field imaging of a tungsten-carbide film by scanning nano-SQUID microscope
}

\author{
Yusuke Shibata and Shintaro Nomura* \\ Division of Physics, University of Tsukuba, \\ Tennodai, Tsukuba, 305-8571, Japan \\ Ryosuke Ishiguro \\ Center for Emergent Matter Science, RIKEN, \\ 2-1 Hirosawa, Wako, Saitama 351-0198, \\ Japan and Department of Mathematical and Physical Sciences, \\ Faculty of Science, Japan Women's University, \\ Mejirodai, Bunkyo-ku, Tokyo 112-8681, Japan \\ Hiromi Kashiwaya and Satoshi Kashiwaya \\ National Institute of Advanced Industrial Science and Technology (AIST), \\ Umezono, Tsukuba, 305-8568, Japan \\ Yusuke Nago and Hideaki Takayanagi \\ Department of Applied Physics, Faculty of Science, \\ Tokyo University of Science, Tokyo 125-8585, Japan
}

(Dated: July 15, 2016)

\begin{abstract}
We present results of magnetic field imaging by scanning nano-superconducting quantum interference device (SQUID) microscopy on a tungsten-carbide (W-C) film fabricated using focusedion-beam chemical vapor deposition. We have investigated magnetic field change by a W-C film in an external magnetic field using a scanning nano-SQUID microscope system. We have found that the reduction of magnetic field above the $\mathrm{W}-\mathrm{C}$ film was $0.9 \%$, indicating penetration of vortices in the $\mathrm{W}-\mathrm{C}$ at an external magnetic field of $0.171 \mathrm{mT}$.
\end{abstract}

*nomura.shintaro.ge@u.tsukuba.ac.jp 


\section{INTRODUCTION}

Focused-ion-beam chemical vapor deposition (FIB-CVD) [1 $[3]$ with $\mathrm{Ga}^{+}$beam and tungsten hexacarboxyl $\left(\mathrm{W}(\mathrm{CO})_{6}\right)$ precursor [4, 5] is a promising technique to prepare superconducting films. FIB-CVD deposition of superconducting films has been acquired much interest as a template-free method to fabricate nano-superconducting quantum interference devices (SQUIDs) that enables precise control of deposition, for example, at the apex of a probe tip of a scanning nano-SQUID microscope. A superconductor/normal metal/superconductor Josephson junction was fabricated using FIB-CVD and Fraunhofer oscillation of the critical current was observed [6]. FIB-CVD also provides a method to repair or modify superconducting circuits [7].

Thin films prepared by FIB-CVD of $\mathrm{W}(\mathrm{CO})_{6}$ are amorphous with typical atomic concentrations of $\mathrm{W}: \mathrm{C}: \mathrm{Ga}=40 \%: 40 \%: 20 \%,[4,5]$ and are often called as tungsten-carbide (W-C) films. W-C nanowires were reported to exhibit superconductivity at a critical temperature $T_{\mathrm{c}}$ of $5.2 \mathrm{~K}$ [4], which is much higher than the critical temperature of bulk crystalline tungsten of $0.01 \mathrm{~K}$ [8]. High $T_{\mathrm{c}}$ of $\mathrm{W}-\mathrm{C}$ nanowires is associated with their amorphous structure [4, 9]. It has been shown that $T_{\mathrm{c}}$ of $\mathrm{W}-\mathrm{C}$ wires was fitted well by a theory as a function of a parameter for the level of disorders [10,11]. Superconductivity of W-C nanowires critically depends on the concentration of $\mathrm{W}$. It was reported that $\mathrm{W}-\mathrm{C}$ nanowires were superconducting for $\mathrm{W}$ concentration between 17.5 and $40 \%$ [11]. A tunneling spectroscopy measurement indicates that a W-C film has a superconducting gap of $\Delta=0.66 \mathrm{meV}$ following s-wave BCS theory [12], while the melting transition in a vortex lattice was observed below $T_{\mathrm{c}}$ by scanning tunneling spectroscopy. [13] The current-voltage properties of W-C strips in high magnetic fields were reported to follow the quasi-three-dimensional vortex glass to liquid transition theory [14].

FIB-CVD deposition of $\mathrm{W}-\mathrm{C}$ films is a promising method for fabrications of nanometersize Josephson junctions and nano-SQUIDs [15]. For this purpose, investigations of diamagnetic and superconductivity properties of $\mathrm{W}-\mathrm{C}$ films are important. We employ a $\mathrm{Nb}$ weak-link scanning nano-SQUID microscope [16] to explore superconducting properties of a W-C film. SQUIDs have been recognized to be the most sensitive magnetic field and flux detectors [17-21]. Efforts have recently been made to reduce the size of the SQUID loop to explore properties of nanoscale objects with high spatial resolution [16, 22 25]. Reduction 
in the size of the SQUID loop allows strong coupling of the SQUID loop to the sample nanoscale object, leading to higher spatial resolution and sensitivities. Moreover, the limiting flux noise spectral density decreases with reduction in the size of the SQUID loop [18] and the magnetic moment sensitivity is approaching single Bohr magneton $\mu_{\mathrm{B}}$ [24]. Recently, we have developed a nano-SQUID microscope based on weak-link Josephson junctions with sufficiently-small hysteresis that enables the SQUID to be operated as a magnetic flux to voltage transducer [16]. In this paper, we investigate magnetic field distribution in the vicinity of a $\mathrm{W}$-C film in an external magnetic field by our Nb weak-link scanning nano-SQUID microscope.

\section{METHODS}

We used a dual-beam FIB-scanning electron microscope system (NB5000, Hitachi HighTechnologies) to deposit W-C films. The acceleration voltage of $\mathrm{Ga}^{+}$ion beam was 40 $\mathrm{kV}$ and the base pressure was $1 \times 10^{-4} \mathrm{~Pa}$. We used tungsten hexacarboxyl $\left(\mathrm{W}(\mathrm{CO})_{6}\right)$ precursor. W-C films with thickness of $300 \mathrm{~nm}$ were grown with $\mathrm{Ga}^{+}$ion beam current of $700 \mathrm{pA}$ at $1 \times 10^{-3} \mathrm{~Pa}$ on a $p$-Si substrate. $\mathrm{Nb} / \mathrm{Au}$ electrodes were fabricated by electron beam lithography with thicknesses of $\mathrm{Nb}$ and $\mathrm{Au}$ of 600 and $30 \mathrm{~nm}$, respectively. Figure 1 shows an optical micrograph of a deposited square W-C film with the size of $20 \mu \mathrm{m} \times 20$ $\mu \mathrm{m}$. On the left side of the square $\mathrm{W}-\mathrm{C}$ film, two $\mathrm{Nb} / \mathrm{Au}$ wires with a width of $2 \mu \mathrm{m}$ were deposited. In the vicinity of the $\mathrm{W}-\mathrm{C}$ film, a yellow blurred region is seen in figure 1 , due to unintentional deposition of $\mathrm{W}-\mathrm{C}$ by the scatterings of ion beams. A W-C strip with the size of $60 \mu \mathrm{m} \times 4 \mu \mathrm{m}$ was prepared for four-terminal electric transport measurements.

We used a home-built scanning nano-SQUID microscope system to measure real-space imaging of magnetic field [16]. A Dayem Nb nano-SQUID probe with a loop size of $1 \mu \mathrm{m}$ and a nano-bridge width of $80 \mathrm{~nm}$ was attached to a quartz tuning fork for a feed-back control of the distance between the sample surface and the SQUID probe. The nanoSQUID probe faced $\theta=51^{\circ}$ to the surface of the sample, and detected magnetic field of $B_{M}(x, y, z)=B_{y}(x, y, z) \sin \theta+B_{z}(x, y, z) \cos \theta$. The sample $\mathrm{W}$-C film was scanned using closed loop inertially-actuated triaxial stepping piezoelectric-stages with resistive position encoders in a cryogen-free pulse tube refrigerator (Optistat PT, Oxford Instruments) with base temperature of $3.4 \mathrm{~K}$. Typical minimum step size was $10 \mathrm{~nm}$ in the coarse positioning 
mode. Scanning measurements were performed at a constant height mode by monitoring the resistive position encoder. Perpendicular magnetic field to the sample surface was applied using a home-made superconducting magnet with the bore size of $50 \mathrm{~mm}$. Details of the scanning nano-SQUID system can be found elsewhere [16]. Electric transport measurements were performed by using a physical property measurement system (PPMS, Quantum Design).

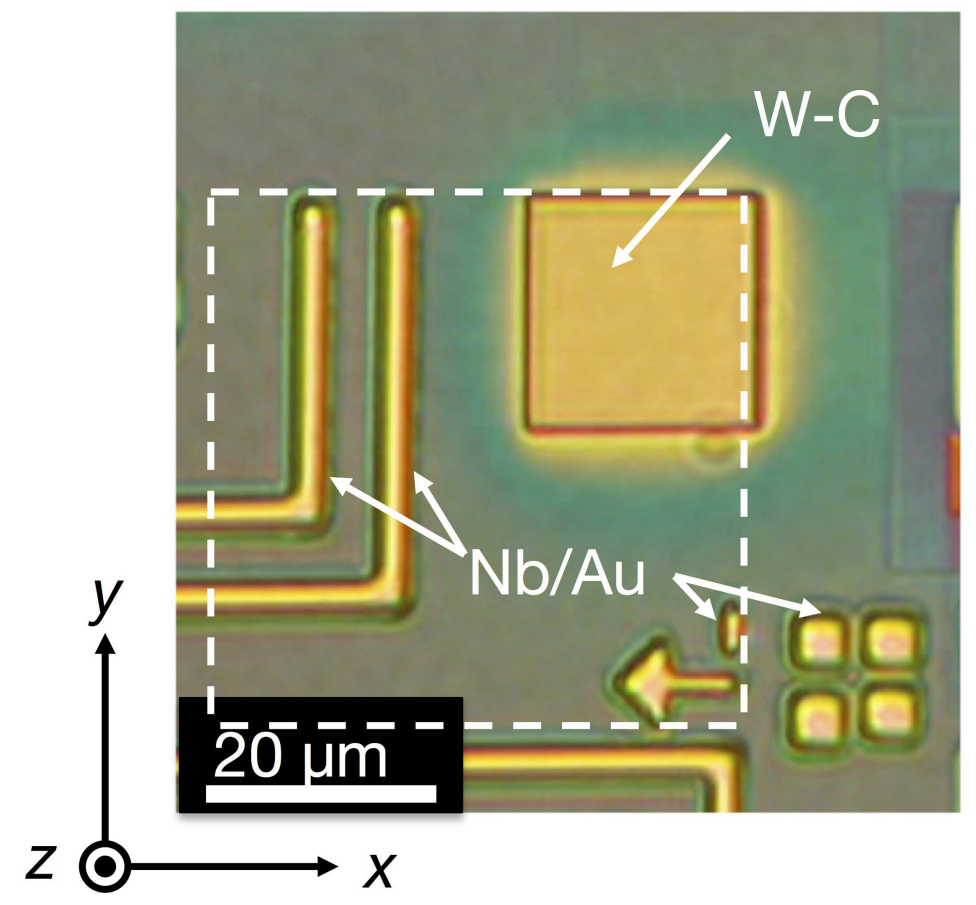

FIG. 1. Optical micropraph of an FIB assisted tungsten carbide film. The thickness and the size of the tungsten carbide film was $300 \mathrm{~nm}$ and $20 \mu \mathrm{m} \times 20 \mu \mathrm{m}$, respectively. Two niobium wires are seen on the left part of the optical micrograph. Dashed white box indicates the displayed area of $45 \mu \mathrm{m} \times 45 \mu \mathrm{m}$ in figure $3(\mathrm{a})$. 
(a)
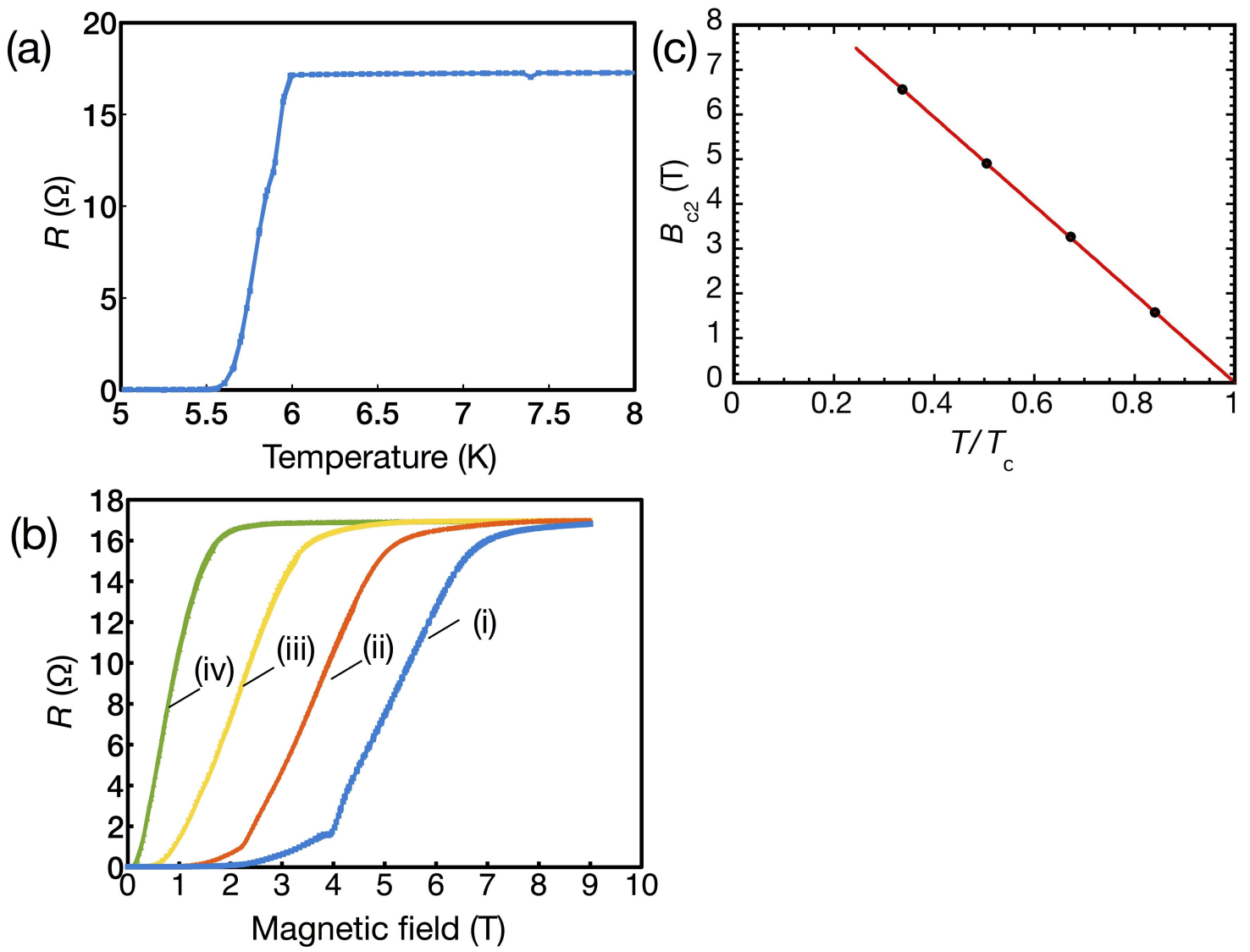

FIG. 2. (a) Temperature dependence of resistance of tungsten-carbide wire at 0 T. (b) Perpendicular magnetic field dependence of resistance of tungsten wire at (i) 2.0, (ii) 3.0, (iii) 4.0 and (iv) $5.0 \mathrm{~K}$. (c) Upper critical field $B_{\mathrm{c} 2}$ as a function of $T / T_{\mathrm{c}}$. The red line is the best fitted line to Eq. (1). 


\section{RESULTS AND DISCUSSION}

\section{A. Characterizations of W-C film}

An optical micrograph of a $\mathrm{W}-\mathrm{C}$ film is shown in figure 1 . The deposited $\mathrm{W}-\mathrm{C}$ is seen to spread about several $\mu \mathrm{m}$ outside of the designed pattern of a square of $20 \mu \mathrm{m} \times 20 \mu \mathrm{m}$. This contrasts with the sharp edges of $\mathrm{Nb} / \mathrm{Au}$ wires fabricated by EB lithography as seen in the left of the square $\mathrm{W}-\mathrm{C}$ in figure 1. Temperature dependence of the four-terminal resistance $(R)$ of the $\mathrm{W}-\mathrm{C}$ wire sample with a width of $4 \mu \mathrm{m}$ is shown in figure 2 (a) at an excitation current of $10 \mu \mathrm{A}$ at $0 \mathrm{~T}$. Figure 2(a) indicates that the $\mathrm{W}$-C sample is zero resistance below $5.5 \mathrm{~K}$. The superconducting transition temperature $T_{\mathrm{c}}$ as defined by $10 \%$ of resistance drop from the resistance at the normal phase $R_{\mathrm{n}}\left(R / R_{\mathrm{n}}=0.9\right)$ is $5.9 \mathrm{~K}$, which is comparable to the reported values of $T_{\mathrm{c}}=4.8-6.2 \mathrm{~K}$ for $\mathrm{W}-\mathrm{C}$ samples fabricated by FIB deposition [4, 5, 11, 12, 14]. The critical current density was estimated to be $J_{c}=1.3 \times 10^{6}$ and $2.1 \times 10^{5} \mathrm{~A} / \mathrm{cm}^{2}$ at 2.0 and $4.0 \mathrm{~K}$, respectively, which is slightly larger than the reported value for thin film of $1.5 \times 10^{5} \mathrm{~A} / \mathrm{cm}^{2}$ at $3 \mathrm{~K}$ [4] probably because of differences in the film thickness and the growth condition.

Figure 2(b) shows perpendicular magnetic field dependence of resistance of the W-C wire sample at 2.0-5.0 K. The upper critical field $B_{\mathrm{c} 2}$ is plotted in figure 2(c) as a function of $T / T_{\mathrm{c}}$. The Ginzburg-Landau coherence length [26] is estimated to be $\xi_{\mathrm{GL}}(0)=5.8 \mathrm{~nm}$ by using

$$
B_{\mathrm{c} 2}=\frac{\Phi_{0}}{2 \pi \xi_{\mathrm{GL}}(0)^{2}}\left(1-\frac{T}{T_{c}}\right)
$$

where $\Phi_{0}$ is the magnetic flux quantum.

\section{B. Mapping of magnetic field change by W-C film}

The scanning nano-SQUID microscopy was performed at a constant height mode with the distance between the SQUID probe and the sample surface of $3.5 \mu \mathrm{m}$. The scanning direction was parallel to the $y$-axis. Mapping of magnetic field normalized by magnetic field of $0.171 \mathrm{mT}$ is shown in figure 3(a). Reduction of magnetic field due to Meissnner effect of the superconducting niobium wire with a width of about $2 \mu \mathrm{m}$ is clearly seen, however, the reduction of magnetic field on the square $\mathrm{W}$-C film of $20 \mu \mathrm{m} \times 20 \mu \mathrm{m}$ is small as shown in 
figure 3(a). A line profile of magnetic field change in figure 3(b) shows reduction of magnetic field due to niobium wires in the region $-17<x<-13 \mu \mathrm{m}$ and at $-11<x<-7 \mu \mathrm{m}$. Reduction of magnetic field due to the $\mathrm{W}-\mathrm{C}$ film was $0.9 \%$.

\section{Calculation of magnetic field distribution by a finite element method}

Magnetic field distributions around $\mathrm{Nb}$ and $\mathrm{W}-\mathrm{C}$ films were calculated by numerically solving Maxwell's and London equations using a finite element method with a commercial package, COMSOL. Nb wires with $2 \mu \mathrm{m} \times 20 \mu \mathrm{m} \times 0.6 \mu \mathrm{m}$ and a W-C film with $20 \mu \mathrm{m} \times$

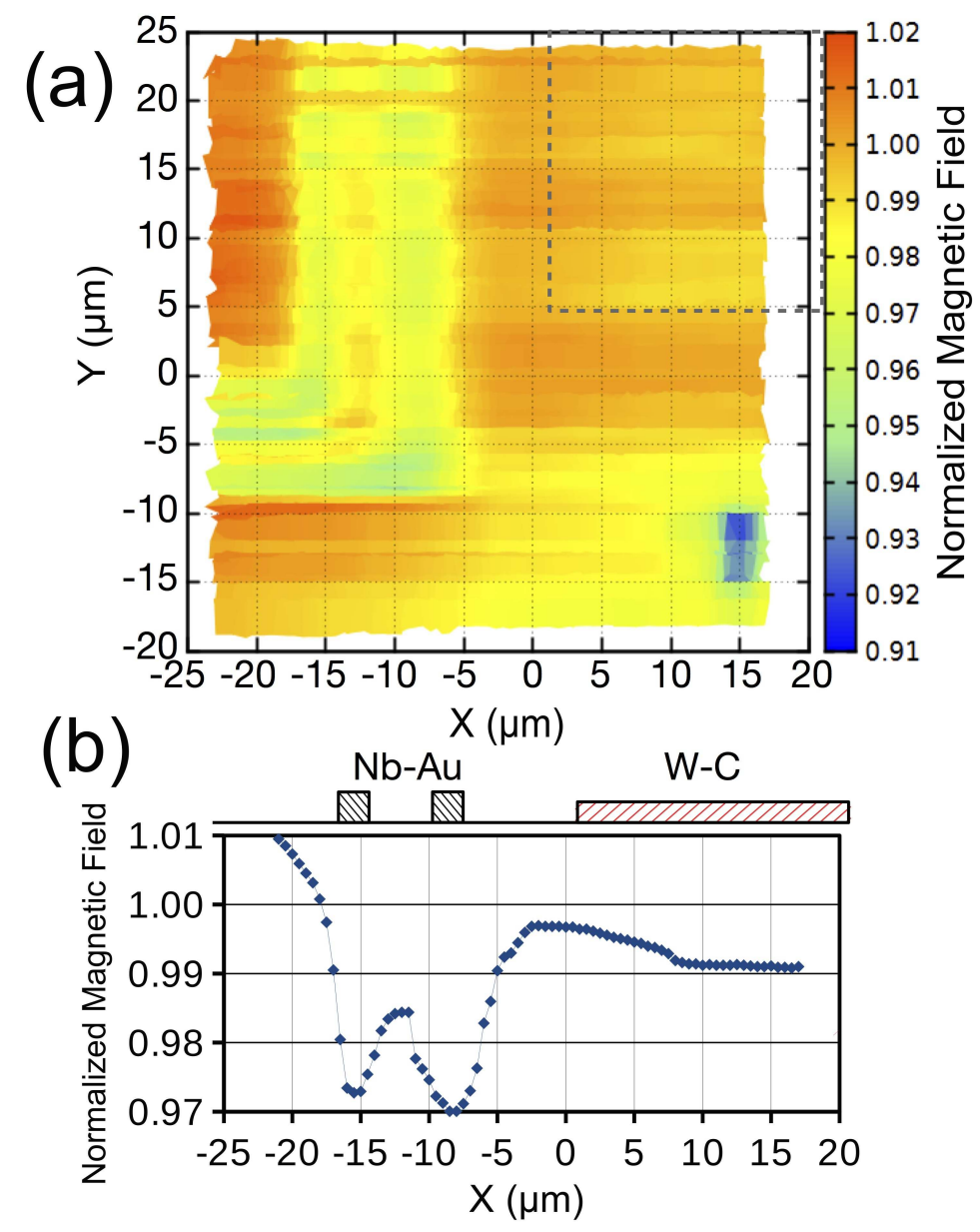

FIG. 3. (a) Mapping of magnetic field normalized by magnetic field of $0.171 \mathrm{mT}$ at $(x, y)=(0 \mu \mathrm{m}$, $0 \mu \mathrm{m})$. The dashed square indicates the position of the tungsten carbide film. (b) A lineprofile of normalized magnetic field averaged at $6 \leq y \leq 10 \mu \mathrm{m}$. The hatched squares on top of the panel indicate the positions of $\mathrm{Nb} / \mathrm{Au}$ and $\mathrm{W}-\mathrm{C}$ films. 
$20 \mu \mathrm{m} \times 0.3 \mu \mathrm{m}$ were modelled in the calculation. London equation $\Delta \mathbf{B}=\mathbf{B} / \lambda_{\text {eff }}^{2}$ was solved inside the $\mathrm{Nb}$ wires and the $\mathrm{W}-\mathrm{C}$ film and Laplace's equation $\Delta \mathbf{B}=0$ was solved elsewhere. The effective London penetration depth $\lambda_{\text {eff }}$ for $\mathrm{Nb}$ wires was fixed to be $1 \mu \mathrm{m}$, and $\lambda_{\text {eff }}$ for a W-C film was varied between 0.85 and $1.6 \mu \mathrm{m}$. Numerical calculations were performed using 1,167,697 domain elements, generated by COMSOL for 3-dimensional region of $80 \times 60 \times 16$ $\mu \mathrm{m}^{3}$ as shown in figure 4 . The sizes of the elements around the $\mathrm{Nb}$ wires and the $\mathrm{W}-\mathrm{C}$ film were approximately 0.042 and $0.17 \mu \mathrm{m}$, respectively.

Figures 5(a) and (b) show calculated perpendicular magnetic field distribution $\left(B_{z}\right)$ on the surface of Si substrate and a crosssection at $y=0 \mu \mathrm{m}$ and $z=3.5 \mu \mathrm{m}$ at an external magnetic field of $0.171 \mathrm{mT}$. Figures $5(\mathrm{a})$ and (b) indicate that the reduction of the magnetic field is larger in the region above the $\mathrm{W}$-C film than in the region above $\mathrm{Nb}$ wires, contradicting with our observations in figure 3 . This result suggests that $\lambda_{\text {eff }}$ much larger than $1.6 \mu \mathrm{m}$ for a W-C film should be assumed to reproduce our result, or alternatively vortices are penetrating through the W-C film. $\lambda_{\text {eff }}$ and $\xi_{\mathrm{GL}}(0)$ for a W-C film with a thickness of 200 $\mathrm{nm}$ was estimated to be 850 and $6.25 \mathrm{~nm}$, respectively [13]. It is unlikely to assume $\lambda_{\text {eff }}$ much larger than $1.6 \mu \mathrm{m}$ because $T_{c}=5.9 \mathrm{~K}$ of our sample is larger than $T_{c}=4.15 \mathrm{~K}$ in the literature [13]. The external magnetic field of $0.171 \mathrm{mT}$ is strong enough to allow penetration of vortices into the $\mathrm{W}$-C film because we have $\Phi / \Phi_{0}=33.1$, where $\Phi=B A$ is the magnetic flux penetrating an area $A=20 \times 20 \mu \mathrm{m}^{2}$. Consequently, we consider the latter is more plausible, and calculated magnetic field distributions around $\mathrm{Nb}$ and $\mathrm{W}-\mathrm{C}$ films by

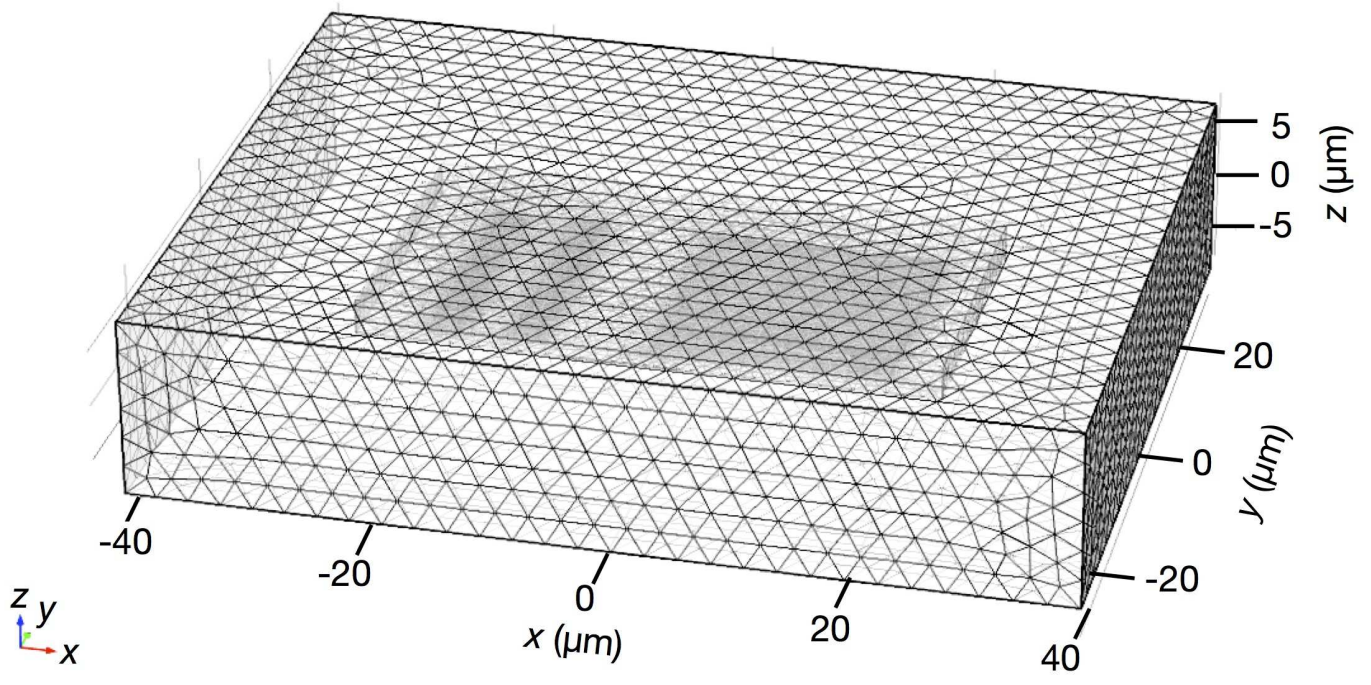

FIG. 4. 3-dimensional meshes for modelling $\mathrm{Nb}$ and $\mathrm{W}-\mathrm{C}$ superconductors. 
assuming 9 vortices are present in the square $20 \mu \mathrm{m} \times 20 \mu \mathrm{m} \mathrm{W}-\mathrm{C}$ film as shown in figures 5(c) and (d). It has been shown by solving a linearlized Ginzburg-Landau equation [27, 28] that the Ginzburg-Landau free energy is lower for a square lattice of vortices than for a triangular lattice due to the square shape of the W-C film [29]. The lineprofiles in figure $5(\mathrm{~d})$ for $\lambda_{\text {eff }}=1.4 \mu \mathrm{m}$ reproduces smaller reduction of the magnetic field on the W-C film than on the Nb wires. This result qualitatively reproduces our observation overlaid in figure $5(\mathrm{~d})$.

Guillamon et al. performed low temperature scanning tunneling microscopy (STM) [12, 13] and spectroscopy measurements in W-C thin films, and they observed absence of hexagonal vortex lattice below about $0.2 \mathrm{~T}$, instead they observed bunching of vortices close to linear depressions observed in a topographic STM image. The spacing between linear de-

(a)

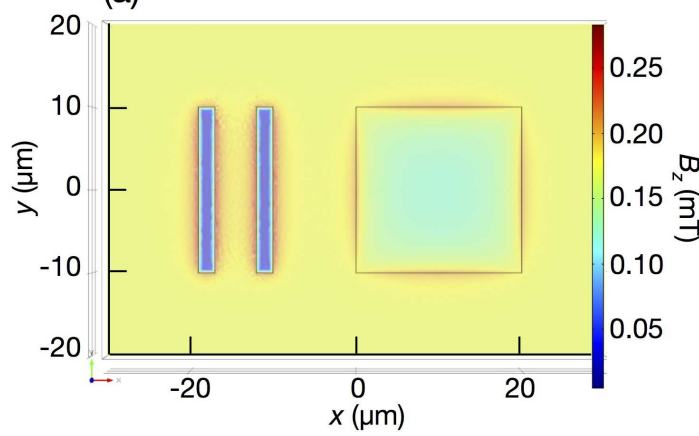

(c)

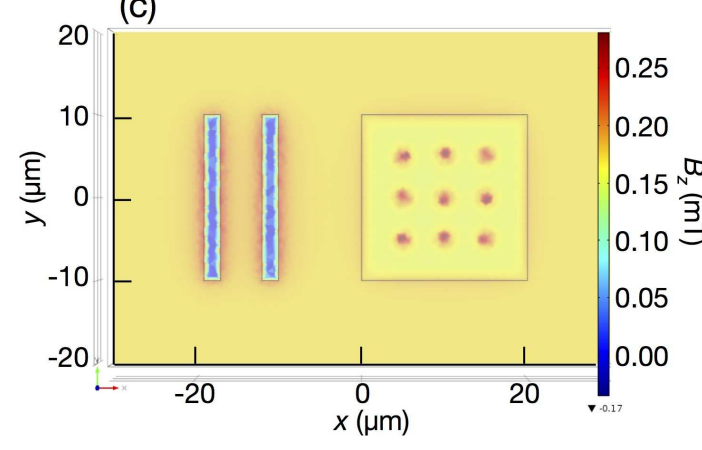

(b) 0.18

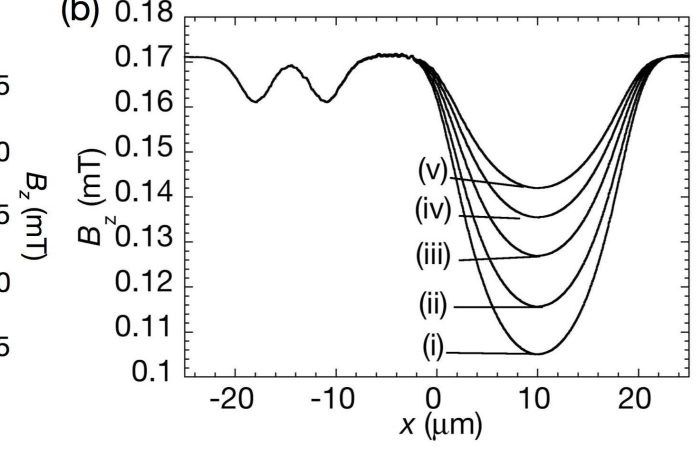

(d)

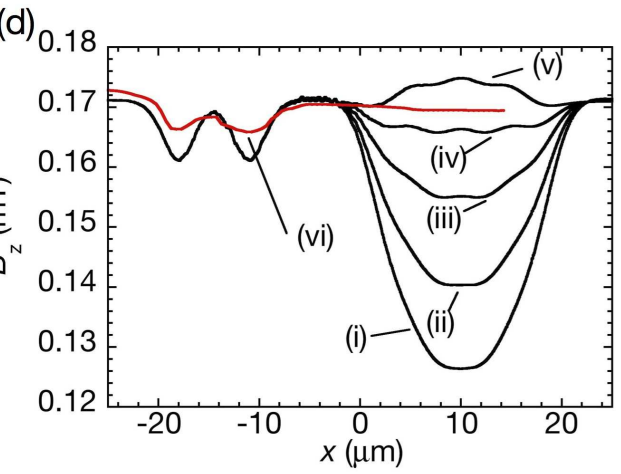

FIG. 5. (a) Calculated perpendicular magnetic field distribution $\left(B_{z}\right)$ on the surface of the sample $(z=0 \mu \mathrm{m})$. (b) Calculated perpendicular magnetic field distribution at $y=0 \mu \mathrm{m}$ and $z=3.5 \mu \mathrm{m}$ for $\lambda_{\text {eff }}=$ (i) 0.85 , (ii) 1.0 , (iii) 1.2 , (iv) 1.4 , and (v) $1.6 \mu \mathrm{m}$. (c) (a) for the case of 9 vortices in the W-C sample. (d) (b) for the case of 9 vortices in the W-C sample. (vi) A line profile of measured magnetic field (red) is also shown. 
pressions was 100-300 nm, below the spatial resolution of our SQUID microscope. Moreover, they found that vortices at smooth and very flat regions change their position easily. The incomplete cancellation of the external magnetic field in the $\mathrm{W}-\mathrm{C}$ film in our measurements is consistent with their observations.

\section{CONCLUSION}

In this paper, reduction of magnetic field on $\mathrm{Nb} / \mathrm{Au}$ wires and a $\mathrm{W}-\mathrm{C}$ film has been measured. We have found that the reduction of magnetic field above the W-C film was $0.9 \%$. The numerically calculated magnetic field distribution with vortices penetrating the W-C film qualitatively reproduces the measured magnetic field change. Our results indicate that FIB CVD deposition of superconducting films is a promising template-free method to fabricate superconducting nano-devices such as nano-SQUIDs.

\section{ACKNOWLEDGEMENTS}

We acknowledge Dr. Yasunori Mawatari for discussions. This work was partly supported by a Grant-in Aid for Scientific Research on Innovative Areas "Topological Material Science" (Nos. 15H05853, 16H00978), and Grant-in-Aid for Scientific Research (Nos. 26610079, 15H03673) from Japan Society for the Promotion of Science. S.N. acknowledges the stimu-

lated discussion in the meeting of the Cooperative Research Project of the Research Institute of Electrical Communication, Tohoku University. 
[1] K. Gamo, N. Takakura, N. Samoto, R. Shimizu, and S. Namba, Jpn. J. Appl. Phys. 23, L293 (1984)

[2] S. Matsui and Y. Ochiai, Nanotechnology 7, 247 (1996)

[3] I. Utke, P. Hoffmann, and J. Melngailis, J. Vac. Sci. Technol. B 26, 1197 (2008)

[4] E. S. Sadki, S. Ooi, and K. Hirata, Appl. Phys. Lett. 85, 6206 (2004)

[5] E. Sadki, S. Ooi, and K. Hirata, Physica C: Superconductivity 426-431, 547 (2005)

[6] J. Dai, R. Kometani, K. Onomitsu, Y. Krockenberger, H. Yamaguchi, S. Ishihara, and S. Warisawa, J. Micromech. Microeng. 24, 055015 (2014)

[7] M. J. Martínez-Pérez, J. Sesé, F. Luis, R. Córdoba, D. Drung, T. Schurig, E. Bellido, R. d. Miguel, C. Gómez-Moreno, A. Lostao, and D. Ruíz-Molina, IEEE Trans. Appl. Super. 11, 345 (2011)

[8] J. W. Gibson and R. A. Hein, Phys. Rev. Lett. 12, 688 (1964)

[9] I. Luxmoore, I. Ross, A. Cullis, P. Fry, J. Orr, P. Buckle, and J. Jefferson, Thin Solid Films 515, 6791 (2007)

[10] M. S. Osofsky, R. J. Soulen, J. H. Claassen, G. Trotter, H. Kim, and J. S. Horwitz, Phys. Rev. Lett. 87, 197004 (2001)

[11] J. Dai, K. Onomitsu, R. Kometani, Y. Krockenberger, H. Yamaguchi, S. Ishihara, and S. Warisawa, Jpn. J. Appl. Phys. 52, 075001 (2013)

[12] I. Guillamón, H. Suderow, S. Vieira, A. Fernández-Pacheco, J. Sesé, R. Córdoba, J. De Teresa, and M. Ibarra, New J. Phys. 10, 093005 (2008)

[13] I. Guillamón, H. Suderow, A. Fernandez-Pacheco, J. Sesé, R. Córdoba, J. M. De Teresa, M. R. Ibarra, and S. Vieira, Nat. Phys. 5, 651 (2009)

[14] Y. Sun, J. Wang, W. Zhao, M. Tian, M. Singh, and M. H. W. Chan, Sci. Rep. 3, 2307 (2013)

[15] C. Granata and A. Vettoliere, Physics Reports 614, 1 (2016)

[16] Y. Shibata, S. Nomura, H. Kashiwaya, S. Kashiwaya, R. Ishiguro, and H. Takayanagi, Sci. Rep. 5, 15097 (2015)

[17] K. Hasselbach, C. Veauvy, and D. Mailly, Physica C 332, 140 (2000)

[18] J. Gallop, Supercond. Sci. Technol. 16, 1575 (2003) 
[19] M. Faley, K. Pratt, R. Reineman, D. Schurig, S. Gott, C. Atwood, R. Sarwinski, D. Paulson, T. Starr, and R. Fagaly, Supercond. Sci. Technol. 17, S324 (2004)

[20] M. E. Huber, N. C. Koshnick, H. Bluhm, L. J. Archuleta, T. Azua, P. G. Bjrnsson, B. W. Gardner, S. T. Halloran, E. A. Lucero, and K. A. Moler, Rev. Sci. Instrum. 79 (2008)

[21] S. Etaki, M. Poot, I. Mahboob, K. Onomitsu, H. Yamaguchi, and H. S. J. van der Zant, Nat. Phys. 4, 785 (2008)

[22] W. Wernsdorfer, Supercond. Sci. Technol. 22, 064013 (2009)

[23] T. Matsumoto, H. Kashiwaya, H. Shibata, H. Takayanagi, S. Nomura, and S. Kashiwaya, Physica C 471, 1246 (2011)

[24] D. Vasyukov, Y. Anahory, L. Embon, D. Halbertal, J. Cuppens, L. Neeman, A. Finkler, Y. Segev, Y. Myasoedov, M. L. Rappaport, M. E. Huber, and E. Zeldov, Nat. Nano. 9, 639 (2013)

[25] D. C. Cox, J. C. Gallop, and L. Hao, Nanofabrication 1, 53 (2014)

[26] M. Tinkham, Introduction to superconductivity, 2nd ed. (McGraw-Hill, 1996)

[27] L. F. Chibotaru, A. Ceulemans, V. Bruyndoncx, and V. V. Moshchalkov, Nature 408, 833 (2000)

[28] T. Mertelj and V. V. Kabanov, Phys. Rev. B 67, 134527 (2003)

[29] Y. Mawatari and S. Kashiwaya, private communications. Supplementary data are available online. 